\title{
Scaling Hypothesis for Matrix Product States
}

\author{
Bram Vanhecke, Jutho Haegeman, Karel Van Acoleyen, Laurens Vanderstraeten, and Frank Verstraete \\ Department of Physics and Astronomy, University of Ghent, Krijgslaan 281, 9000 Gent, Belgium
}

(Received 12 September 2019; published 18 December 2019)

\begin{abstract}
We study critical spin systems and field theories using matrix product states, and formulate a scaling hypothesis in terms of operators, eigenvalues of the transfer matrix, and lattice spacing in the case of field theories. The critical point, exponents, and central charge are determined by optimizing them to obtain a data collapse. We benchmark this method by studying critical Ising and Potts models, where we also obtain a scaling Ansatz for the correlation length and entanglement entropy. The formulation of those scaling functions turns out to be crucial for studying critical quantum field theories on the lattice. For the case of $\lambda \phi^{4}$ with mass parameter $\mu^{2}$ and lattice spacing $a$, we demonstrate a double data collapse for the correlation length $\delta \xi(\mu, \lambda, D)=\tilde{\xi}\left(\left(\alpha-\alpha_{c}\right)(\delta / a)^{-1 / \nu}\right)$ with $D$ the bond dimension, $\delta$ the gap between eigenvalues of the transfer matrix, and $\alpha_{c}=\mu_{R}^{2} / \lambda$ the parameter which fixes the critical quantum field theory.
\end{abstract}

DOI: 10.1103/PhysRevLett.123.250604

Introduction.-Traditional numerical techniques for simulating extensive many-body systems such as Monte Carlo sampling (MC) and exact diagonalization (ED) naturally come with a dimensionful parameter that controls the level of approximation: the system size $L$. One of the major insights that has allowed the simulation of systems at or near criticality has been the realization that, rather than simply pushing $L \rightarrow \infty$, the numerical results at different system sizes can be combined using the concept of finitesize scaling [1-3], changing the finite $L$ from a weakness to a strength. The crucial idea is that $1 / L$ acts as a relevant perturbation, and thus enters in the singular part of the free energy. By invoking the scaling hypothesis, the latter is a generalized homogeneous function

$$
f\left(\left\{t_{i}\right\}, 1 / L\right)=s^{-d} f\left(\left\{s^{\alpha_{i}} t_{i}\right\}, s / L\right),
$$

where $s$ is a free parameter that parametrizes a scale transformation, $\left\{t_{i}\right\}$ is the set of coupling constants to (relevant) perturbations in the theory, and $\left\{\alpha_{i}\right\}$ are their corresponding scaling dimensions. This scaling hypothesis can then be used to perform a collapse of numerical datasets for different $L$ and thereby obtain accurate estimates for critical exponents and the location of a critical point. Furthermore, in the context of quantum field theory (QFT) lattice simulations, finite-size scaling ideas have proven vital for reaching the continuum limit $[4,5]$.

Here we consider the application of matrix product states (MPS) methods [6-9] for simulating critical 1D quantum or 2D classical spin systems, including the continuum limit of lattice descriptions for QFTs. Results extracted from MPS calculations are affected by the finite bond dimension $D$ of the matrices, which is in fact a proxy for the finite amount of entanglement in the simulated state. The traditional approach to study phase transitions, and, relatedly, continuum limits of lattice field theories, with, e.g., the (finite) density matrix renormalization group algorithm [6] has been to extrapolate predictions for one or more order parameters $M^{\prime}=\lim _{D \rightarrow \infty} M(D)$ towards the limit of infinite bond dimension $D$. A different approach emerged from the realization that the finite bond dimension acts as a relevant perturbation, similar to the finite system size, and induces an additional length scale in the problem, which results in crossover behavior between the two scales [10,11]. Unlike MC and ED, MPS methods can work directly in the thermodynamic limit and eliminate the finite size scale. At criticality, only the length scale associated with the finite bond dimensions remains, and this was identified as the MPS correlation length $\xi^{(D)}$ (as defined below). This correlation length was shown to scale as $\xi^{(D)} \sim D^{\kappa}$ in the asymptotic limit of large $D$ [12]. Here, a new critical exponent $\kappa$ was introduced, which is a function of the central charge of the associated conformal field theory (CFT) [11,13].

Proper finite-entanglement scaling Ansätze are paramount for the further development of the tensor network framework for simulating (near) critical theories and QFTs. This holds even more for higher dimensional systems simulated with, e.g., projected entangled pair states (PEPS) [14], for which the computational cost grows much faster with increasing bond dimension $D$. The scaling behavior in the discrete variable $D$ only holds for sufficiently large $D$ and cannot be expected to be smooth or homogeneous for small or intermediate values of $D$. Instead, scaling Ansatz can be formulated directly in terms of the finite correlation length $\xi^{(D)}$ [15-18]. However, the (inverse) correlation length $1 / \xi^{(D)}$ only correctly quantifies the strength of the relevant perturbation associated with the 
finite entanglement approximation and tends to zero for $D \rightarrow \infty$, exactly at criticality.

In this Letter, we motivate and introduce the use of entanglement scaling for translation invariant MPS in the thermodynamic limit, based on a different (inverse) length scale $\delta$ defined in terms of the gaps in the full spectrum of (inverse) correlation lengths, as obtained from the (negative) logarithm of the eigenvalues of the transfer matrix [19]. A careful study of the nature of the MPS approximation [19-21] indicates that these gaps are a direct consequence of the finite bond dimension and go to zero for $D \rightarrow \infty$, regardless whether the system is gapped or not. In particular, the correlation length $\xi^{(D)}$ can itself be scaled in terms of $\delta$, as was first illustrated by Rams et al. for gapped systems [22]. Combined with other relevant perturbations, a full scaling Ansatz for the correlation length itself can thus be formulated. We illustrate this for the two-dimensional classical Ising and Potts models. Understanding the resulting scaling functions is also of crucial importance to simulate continuum limits of spin systems in the form of QFTs. The continuum limit is obtained by taking the limit of bond dimension going to infinity and lattice spacing to zero, and we demonstrate that this double scaling limit yields a double data collapse near the critical point of $(2+0)$-dimensional $\lambda \phi^{4}$ theory.

Entanglement scaling hypothesis.-Throughout this Letter, we use uniform MPS which depend on a single tensor to parametrize translation-invariant states directly in the thermodynamic limit. We point the reader to Refs. [23-25] for details on methods that use MPS to study classical spin systems. An MPS provides a variational approximation for low-energy states by truncating in the entanglement spectrum, which has its repercussion on the approximation of the physical properties of the system. In particular, correlation functions are represented by a linear combination of exponentially decaying functions, where the spectrum of inverse correlation lengths $\epsilon_{i}$ is determined by the eigenvalues of the MPS transfer matrix $\lambda_{i}$ as

$\epsilon_{i}=\xi_{i}^{-1}=-\log \left|\lambda_{i}\right|, \quad$ with $\epsilon_{i} \leq \epsilon_{i+1},\left(i=0, \ldots, D^{2}-1\right)$,

assuming the MPS is normalized such that $\epsilon_{0}=0$. The actual correlation length of the state is then identified as $\xi \equiv \xi_{1}$. Close to a second order critical point, however, we expect correlation functions to exhibit a power-law contribution multiplied with the exponential decay, which can be understood from the Källèn-Lehmann representation of correlation functions as a linear combination of a continuum of exponentials [19]. The MPS transfer matrix thus provides a discretized approximation to this continuous spectrum of correlation length, and the spacing between them is a reflection of an inverse system size. Indeed, by interpreting the true state as resulting from an infinite amount of imaginary-time evolution (i.e., the path-integral representation), the discretization of the spectrum of correlation lengths can be understood as resulting from the compression of the infinite imaginary-time interval inherent in the MPS approximation [20,21]. Hence, the gaps in the transfer-matrix spectrum can be related to a finite size in imaginary time.

Therefore we can build a finite-entanglement scaling theory by quantifying the discreteness of the spectrum of inverse correlation lengths, i.e., the gaps in the transfermatrix spectrum. The simplest definition is $\delta=\epsilon_{2}-\epsilon_{1}$, which was used in Ref. [22] to extrapolate the correlation length itself. However, as also remarked in Ref. [22], the spectrum can consist of different sectors and it can be useful to consider a generalized definition

$$
\delta=\sum_{i=1}^{n} c_{i} \epsilon_{i}, \quad \text { with } \quad \sum_{i} c_{i}=0,
$$

with a finite number $n$ sufficiently smaller than $D^{2}$, such that only the smallest eigenvalues $\epsilon_{i}$ are included. For any choice of the coefficients $c_{i}$ such that $\sum_{i} c_{i}=0$, this quantity should converge to zero for $D \rightarrow \infty$. Evidently, the $\epsilon_{i}$, and thus also $\delta$, transform as an inverse length under scale transformations. Therefore, we can formulate the scaling hypothesis for an order parameter $m$, thus

$$
m(t, \delta)=s^{-\beta / \nu} m\left(s^{1 / \nu} t, s \delta\right) .
$$

This yields the expression for a corresponding scaling function $\tilde{m}$

$$
\delta^{-\beta / \nu} m(t, \delta)=m\left(\delta^{-1 / \nu} t, 1\right)=\tilde{m}\left(\delta^{-1 / \nu} t\right) .
$$

From the scaling property of $\delta$, it follows that the scaling functions $\tilde{m}$ away from the origin exhibits a power-law behavior with the correct exponent. However, unlike in traditional finite-size scaling, where the finite size imposes smoothness on the scaling function, the MPS scaling function may be nonanalytic. Around such nonanalycities the scaling function exhibits mean-field exponents, in accordance with Ref. [26].

In a similar vein, we formulate a scaling hypothesis for the correlation length

$$
\delta \xi(t, \delta)=\xi\left(\delta^{-1 / \nu} t, 1\right)=\tilde{\xi}\left(\delta^{-1 / \nu} t\right)
$$

in terms of a scaling function $\tilde{\xi}$. Crucially, this scaling behavior justifies prior approaches where a scaling Ansatz for $m$ was formulated directly in terms of $1 / \xi$ instead of $\delta$. But using $\delta$, which objectively quantifies the perturbation strength due to the finite bond dimension, the ability to also scale the correlation length yields additional data points in order to fit more accurately the location of the critical point and the corresponding scaling exponents.

Finally, we can also extract the bipartite entanglement entropy from the entanglement spectrum of a given MPS. 
From CFT calculations [27], we know that $\exp [(6 / c) S]$ scales as a length, so we can write down a scaling hypothesis of the form

$$
\exp \left(\frac{6}{c} S(t, \delta)\right)=s \exp \left(\frac{6}{c} S\left(s^{-1 / \nu} t, s \delta\right)\right)
$$

where $c$ is the central charge of the CFT associated with the critical point.

For a given set of data points at different MPS bond dimensions, the critical properties of the model can now be determined by optimizing a data collapse in terms of $\delta$. In principle, every $\delta$ built up from a set of $c_{i}$ should give the right scaling behavior, but in order to improve the collapse, the $c_{i}$ can also be treated as parameters. The cost function that we optimize is the sum of the $y$ direction distances of all data points to a scaling function, which is itself parametrized by a set of parameters. We feed this in to a standard nonlinear optimization algorithms for determining these different parameters [28]. Note that there is no consensus on an ultimate algorithm to perform finite size scaling and data collapse, this remains an active area of research [29-33].

Two-dimensional Ising and Potts models.-As a first illustration of our method, we consider the classical Ising model on the square lattice. We have computed a set of MPS with $D$ ranging between 10 and 200, for different temperatures around the critical point $T_{c}=2.269185314$. Here, we fix the $c_{i}$ by hand, defining $\delta=\epsilon_{4}-\epsilon_{2}$, such that we obtain a collapse of the data. In Fig. 1 we plot the scaling functions for the order parameter, correlation length, and entanglement entropy (as they were defined above), using the known values of the critical temperature, the exponents $\nu=1$ and $\beta=1 / 8$, and the central charge $c=1 / 2$. If we jointly optimize the collapse of order parameter and correlation length we find a critical temperature of $T_{c}^{\mathrm{MPS}}=2.269184934$ with critical exponents $\nu=$ 0.99980 and $\beta=0.12534$.

Second, we study the three-state Potts model. We have used MPS with $D$ ranging between 21 and 120, around the critical point $T_{c}=0.994972861$. Here, we have used $\delta=\frac{5}{2} \epsilon_{2}-\epsilon_{4}-\epsilon_{5}-\frac{1}{2} \epsilon_{6}$. In Fig. 2 we plot the three scaling functions, using the known values for the critical data $\nu=$ $5 / 6 \approx 0.833$ and $\beta=1 / 9 \approx 0.111$ and a central charge $c=4 / 5$. If we jointly optimize the collapse of $\xi$ and $m$ for this model, we find $T_{c}=0.9949715, \nu=0.8283$, and $\beta=0.1086$. The extracted critical exponents for both these models were found with similar accuracy to alternative tensor network methods $[34,35]$. Note that for both these models we used a wide range of bond dimensions for illustrating the robustness of the scaling hypothesis. Not surprisingly we find that in general the collapse of the data gets better for larger bond dimensions, similar to finite size collapses that improve for larger system sizes. The guiding principle is therefore to choose bond dimensions as large as possible, while still being spaced wide enough to allow for extracting the scaling in $\delta$.

$\lambda \phi^{4}$ field theory.-Finally, as a more exotic application, we look at a phase transition in a QFT described by the following Lagrangian density of the real field $\phi$ :

$$
\mathcal{L}(\phi)=\frac{1}{2} \partial_{\mu} \phi \partial^{\mu} \phi+\frac{1}{2} \mu_{p}^{2} \phi^{2}+\frac{1}{4} \lambda_{p} \phi^{4} .
$$

The resulting Euclidean path integral can be discretized in a standard way, e.g.: $\partial_{1} \phi(x, y)=\left(\phi_{i+1, j}-\phi_{i, j}\right) / a$ (with $a$ the lattice spacing) and converted into a tensor-network form by truncating the $\phi$ fields in a suitable basis, see Ref. [36]. In order to study the second-order QFT phase transition from the $\mathbb{Z}_{2}$ unbroken phase $\langle\phi\rangle=0$ to the broken phase $\langle\phi\rangle \neq 0$ [37], we computed uniform-MPS approximations of the fixed point of the path-integral transfer operator. In addition to the entanglement scaling $\delta \rightarrow 0$, we also have to consider the continuum scaling $a \rightarrow 0$. Rather than taking both limits separately, which up until now has been the standard procedure for MPS simulations of QFTs [38-40], we will show how one can perform a double collapse on all the results for different lattice spacings and bond dimensions into a single scaling function.
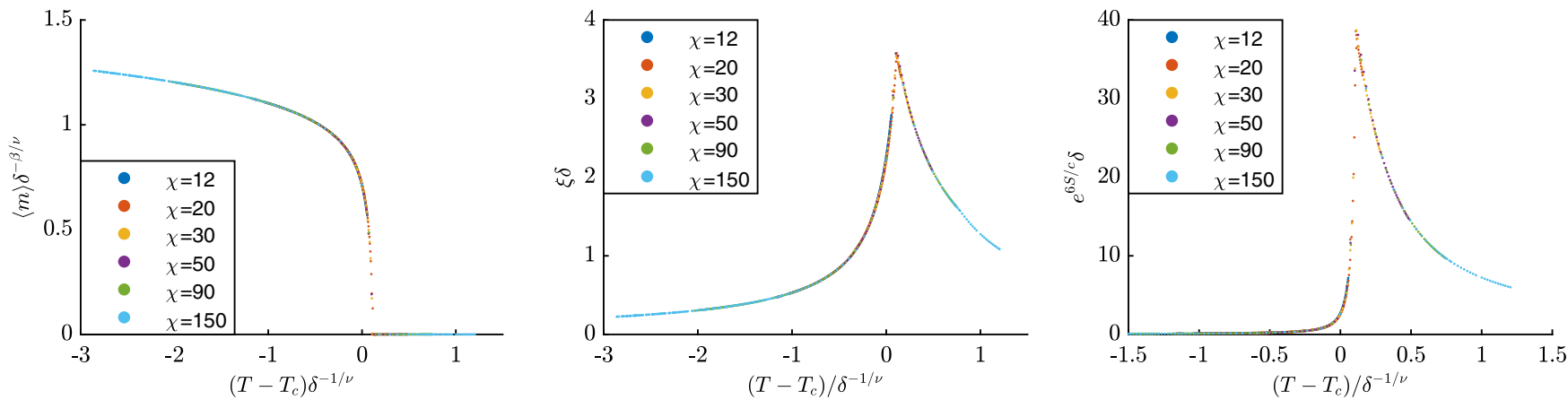

FIG. 1. Collapse plots for the Ising model, calculated with MPS of bond dimension 12,20,30,50,90, and 150, for 189 different temperatures linearly spaced between $T=2.2666$ and $T=2.2698$. Left, magnetization; middle, correlation length; right, bipartite entanglement entropy. 

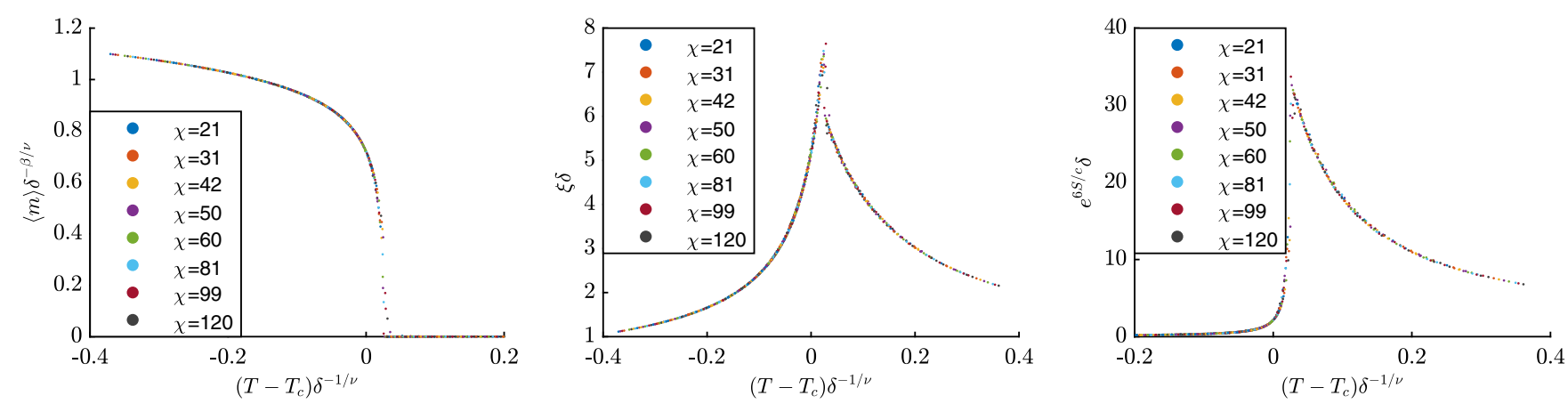

FIG. 2. Collapse plots for the Potts model, calculated with MPS of bond dimension 21,31,42,50,60,81,99, and 120, for 96 different temperatures linearly spaced between $T=0.9939$ and $T=0.9954$. Left, magnetization; middle, correlation length; right, bipartite entanglement entropy.

We first consider the continuum scaling of the Euclidean lattice path integral. The lattice action is defined in terms of the lattice parameters $\lambda=\lambda_{p} a^{2}$ and $\mu^{2}=\mu_{p}^{2} a^{2}$. (We use the subscript $p$ for quantities in physical units, independent of the lattice spacing $a$.) However, in the continuum limit, the mass term receives a divergent one-loop correction, such that the bare mass $\mu_{p}=\mu / a$ of the theory is parametrized in terms of a renormalized mass parameter $\mu_{p, R}=\mu_{R} / a$ as

$$
\mu^{2}=\mu_{R}^{2}-3 \lambda A\left(\mu_{R}^{2}\right),
$$

where the one-loop contribution $A(x)$ is given in, e.g., Ref. [36] and diverges as $\log (x)$ for small values of its argument. The $\lambda \phi^{4}$ theory being superrenormalizable, this is the only UV divergence and the IR behavior is then completely characterized by the finite ratio $\alpha=\mu_{p, R}^{2} / \lambda_{p}=$ $\mu_{R}^{2} / \lambda$. In approaching the continuum limit, both the mass term and interaction term get additional UV-finite corrections. Rather than computing these in perturbation theory, we parametrize general corrections and determine the coefficients as part of the scaling analysis. Specifically, we consider the following parametrization,

$$
\begin{aligned}
\mu^{2} & =\lambda g-3 \lambda A(\lambda g), \\
\alpha & =g+\lambda P(\lambda, g), \\
a^{2} & =\lambda+\lambda^{2} P^{\prime}(\lambda, g),
\end{aligned}
$$

with $g$ a free parameter that tends to $\alpha$ in the continuum limit $\lambda \rightarrow 0$, and where $P$ and $P^{\prime}$ are multivariate polynomials. The existence of a continuum limit then requires that, e.g., the lattice correlation length $\xi(\mu, \lambda, D)$ corresponds to a physical correlation length $\xi_{p}=\xi a$ that is only a function of $\alpha$ and the gap in the physical spectrum of inverse correlation lengths $\delta_{p}=\delta / a$, giving rise to the continuum scaling hypothesis

$$
\xi(\mu, \lambda, D)=\frac{1}{a} \xi_{p}\left(\alpha, \frac{\delta}{a}\right)
$$

with $\mu, \lambda$, and $\delta$ parameters in lattice units.
As the continuum theory exhibits itself a phase transition at $\alpha=\alpha_{c}$, the scaling hypothesis for the field theory requires that $\xi_{p}$, now parametrized in terms of $\Delta \alpha=\alpha-\alpha_{c}$ and $\delta_{p}=\delta / a$ is a generalized homogeneous function

$$
s \xi_{p}\left(\Delta \alpha, \delta_{p}\right)=\xi_{p}\left(s^{-1 / \nu} \Delta \alpha, s^{-1} \delta_{p}\right),
$$

and thus $\delta_{p} \xi_{p}\left(\Delta \alpha, \delta_{p}\right)=\tilde{\xi}\left(\delta_{p}^{-1 / \nu} \Delta \alpha\right)$. Combining the IR scaling hypothesis for the critical field theory with the continuum scaling Ansatz, yields a double collapse for the quantities of the lattice theory

$$
\delta \xi(\mu, \lambda, D)=\tilde{\xi}\left(\left(\frac{\delta}{a}\right)^{-1 / \nu} \Delta \alpha\right)
$$

For the double collapse equation of the order parameter $\langle\phi\rangle$ the steps are very similar, except that now we consider multiplicative corrections to the wave-function renormalization:

$$
\tilde{\phi}\left(\Delta \alpha\left(\frac{\delta}{a}\right)^{-1 / \nu}\right)=\left(\frac{\delta}{a}\right)^{-\beta / \nu}\langle\phi\rangle(\mu, \lambda, D)\left[1+\lambda P^{\prime \prime}(\lambda, g)\right] .
$$

The phase transition in $\lambda \phi^{4}$ field theory has been studied by lattice Monte Carlo simulations [41-43], Hamiltonian truncation [44], and tensor-network methods [36,39,45], where the most accurate estimates $[39,43]$ agree on a value for the critical point $\alpha_{c} \approx 11.05-11.07$. We have generated 701 data points with arbitrary lattice spacing $0.005<a^{2}<0.1$, bond dimensions ranging from 50-150, and couplings $\alpha$ around the critical point. Our scaling approach allows us to transform those 701 data points $(\xi,\langle\phi\rangle, \mu, \lambda, \delta)$ with the best guess of $P, P^{\prime}$, and $P^{\prime \prime}$ to points $(\tilde{\xi}, \tilde{\phi}, \alpha, a, \delta)$, plot them according to the above collapse equations, and compare them to a scaling functions to optimize the fit parameters, essentially the same as we did for the Ising and Potts model. We fixed $\nu=1$ and $\beta=1 / 8$ and optimized a fit using $P$ 's of order 1,2 , and 3 in $\lambda$ and order 3,4 , and 5 in $g$ and found, respectively, $1 / \alpha_{c}=11.06093,1 / \alpha_{c}=11.06072$, and $1 / \alpha_{c}=11.06886$. 


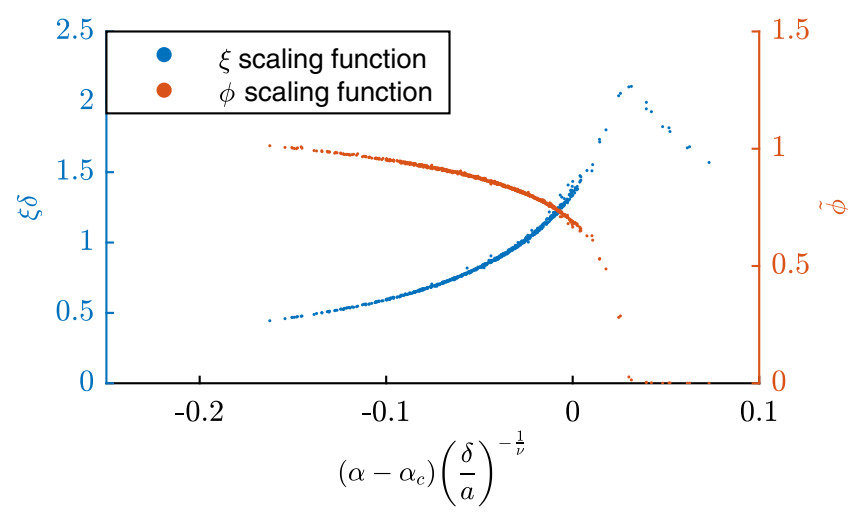

FIG. 3. Double collapse plot of the order parameter and correlation length for $\lambda \phi^{4}$ field theory. The left $y$ axis is associated with the correlation length, the right one with $\langle\phi\rangle$. The used bond dimensions are scattered over the full interval [50, 150].

These should be compared to $1 / \alpha_{c}=10.913(56)$, an alternative tensor network based study of $\lambda \phi^{4}$ [36] and $1 / \alpha_{c}=11.055(14)$, the leading MC study [43]. We show the third order collapse plot in Fig. 3.

Conclusions.-We have formulated a finite scaling hypothesis for MPS based simulations of transfer matrices of classical spin systems near criticality. We have identified a natural analog of the inverse system size $1 / L$ in terms of a scaling parameter $\delta \simeq 1 / L$ that is a function of the eigenvalues of the MPS transfer matrix. The viability of our general scaling hypothesis was demonstrated explicitly on $d=2+0$ Ising, Potts, and $\lambda \phi^{4}$, the latter case requiring a double collapse both in $\delta$ and lattice spacing. An open question here is whether a similar double collapse can be obtained for the case of nonsuperrenormalizable field theories. Notice also that our scaling hypothesis is not specific for partition function methods, similar results would be obtained for quantum Hamiltonians. Finally, going to higher dimensions, for simulating $2+1$ and $3+0$ critical systems with PEPS, similar scaling ideas give rise to two inverse length scales $\delta_{1}$ and $\delta_{2}$. This situation is similar to a system on a $L_{1} \times L_{2} \times \infty$ cuboid, for which we can borrow scaling ideas used in ED and MC, to be reported elsewhere.

This work was made possible through the support of the ERC Grants QUTE (647905), ERQUAF (715861), and QTFLAG.

[1] M. E. Fisher and M. N. Barber, Scaling Theory for Finite-Size Effects in the Critical Region, Phys. Rev. Lett. 28, 1516 (1972).

[2] E. Brézin, An investigation of finite size scaling, J. Phys. II (France) 43, 15 (1982).

[3] J. Cardy, Finite-Size Scaling (Elsevier, 1988).

[4] M. Luscher, P. Weisz, and U. Wolff, A numerical method to compute the running coupling in asymptotically free theories, Nucl. Phys. B359, 221 (1991).
[5] K. Jansen, C. Liu, M. Luscher, H. Simma, S. Sint, R. Sommer, P. Weisz, and U. Wolff, Nonperturbative renormalization of lattice QCD at all scales, Phys. Lett. B 372, 275 (1996).

[6] S. R. White, Density Matrix Formulation for Quantum Renormalization Groups, Phys. Rev. Lett. 69, 2863 (1992).

[7] F. Verstraete, D. Porras, and J. I. Cirac, Density Matrix Renormalization Group and Periodic Boundary Conditions: A Quantum Information Perspective, Phys. Rev. Lett. 93, 227205 (2004).

[8] F. Verstraete, V. Murg, and J. I. Cirac, Matrix product states, projected entangled pair states, and variational renormalization group methods for quantum spin systems, Adv. Phys. 57, 143 (2008).

[9] U. Schollwöck [The density-matrix renormalization group in the age of matrix product states, Ann. Phys. (Amsterdam) 326, 96 (2011)].

[10] T. Nishino, K. Okunishi, and M. Kikuchi, Numerical renormalization group at criticality, Phys. Lett. A 213, 69 (1996).

[11] B. Pirvu, G. Vidal, F. Verstraete, and L. Tagliacozzo, Matrix product states for critical spin chains: Finite-size versus finite-entanglement scaling, Phys. Rev. B 86, 075117 (2012).

[12] L. Tagliacozzo, T. R. de Oliveira, S. Iblisdir, and J. I. Latorre, Scaling of entanglement support for matrix product states, Phys. Rev. B 78, 024410 (2008).

[13] F. Pollmann, S. Mukerjee, A. M. Turner, and J. E. Moore, Theory of Finite-Entanglement Scaling at One-Dimensional Quantum Critical Points, Phys. Rev. Lett. 102, 255701 (2009).

[14] F. Verstraete and J. I. Cirac, Renormalization algorithms for quantum-many body systems in two and higher dimensions, arXiv:cond-mat/0407066.

[15] P. Corboz, P. Czarnik, G. Kapteijns, and L. Tagliacozzo, Finite Correlation Length Scaling with Infinite Projected Entangled-Pair States, Phys. Rev. X 8, 031031 (2018).

[16] M. Rader and A. M. Läuchli, Finite Correlation Length Scaling in Lorentz-Invariant Gapless iPEPS Wave Functions, Phys. Rev. X 8, 031030 (2018).

[17] P. Czarnik and P. Corboz, Finite correlation length scaling with infinite projected entangled pair states at finite temperature, Phys. Rev. B 99, 245107 (2019).

[18] J. C. Pillay and I.P. McCulloch, Cumulants and scaling functions of infinite matrix product states, arXiv: 1906.03833.

[19] V. Zauner, D. Draxler, L. Vanderstraeten, M. Degroote, J. Haegeman, M. M. Rams, V. Stojevic, N. Schuch, and F. Verstraete, Transfer matrices and excitations with matrix product states, New J. Phys. 17, 053002 (2015).

[20] M. M. Rams, V. Zauner, M. Bal, J. Haegeman, and F. Verstraete, Truncating an exact matrix product state for the XY model: Transfer matrix and its renormalization, Phys. Rev. B 92, 235150 (2015).

[21] M. Bal, M. M. Rams, V. Zauner, J. Haegeman, and F. Verstraete, Matrix product state renormalization, Phys. Rev. B 94, 205122 (2016).

[22] M. M. Rams, P. Czarnik, and L. Cincio, Precise Extrapolation of the Correlation Function Asymptotics in Uniform Tensor Network States with Application to the BoseHubbard and XXZ Models, Phys. Rev. X 8, 041033 (2018). 
[23] J. Haegeman and F. Verstraete, Diagonalizing transfer matrices and matrix product operators: A medley of exact and computational methods, Annu. Rev. Condens. Matter Phys. 8, 355 (2017).

[24] M. T. Fishman, L. Vanderstraeten, V. Zauner-Stauber, J. Haegeman, and F. Verstraete, Faster methods for contracting infinite two-dimensional tensor networks, Phys. Rev. B 98, 235148 (2018).

[25] L. Vanderstraeten, J. Haegeman, and F. Verstraete, Tangentspace methods for uniform matrix product states, SciPost Phys. Lect. Notes 7 (2019).

[26] C. Liu, L. Wang, A. W. Sandvik, Y.-C. Su, and Y.-J. Kao, Symmetry breaking and criticality in tensor-product states, Phys. Rev. B 82, 060410(R) (2010).

[27] P. Calabrese and J. Cardy, Entanglement entropy and quantum field theory, J. Stat. Mech. (2004) P06002.

[28] We used the function lsqnonlin.m of matlab with the setting levenberg-marquardt, the scaling function was parametrized such that its behavior far from zero would be power law (as it is known it should), which aided in the convergence.

[29] N. Kawashima and N. Ito, Critical behavior of the threedimensional $\pm \mathrm{j}$ model in a magnetic field, J. Phys. Soc. Jpn. 62, 435 (1993).

[30] S. M. Bhattacharjee and F. Seno, A measure of data collapse for scaling, J. Phys. A 34, 6375 (2001).

[31] J. Houdayer and A. K. Hartmann, Low-temperature behavior of two-dimensional Gaussian Ising spin glasses, Phys. Rev. B 70, 014418 (2004).

[32] S. Wenzel, E. Bittner, W. Janke, and A. M. J. Schakel, Percolation of vortices in the $3 \mathrm{~d}$ Abelian lattice Higgs model, Nucl. Phys. B793, 344 (2008).

[33] F. Winter, W. Janke, and A. M. J. Schakel, Geometric properties of the three-dimensional Ising and XY models, Phys. Rev. E 77, 061108 (2008).
[34] M. Hauru, G. Evenbly, W. Wei Ho, D. Gaiotto, and G. Vidal, Topological conformal defects with tensor networks, Phys. Rev. B 94, 115125 (2016).

[35] G. Evenbly and G. Vidal, Local Scale Transformations on the Lattice with Tensor Network Renormalization, Phys. Rev. Lett. 116, 040401 (2016).

[36] D. Kadoh, Y. Kuramashi, Y. Nakamura, R. Sakai, S. Takeda, and Y. Yoshimura, Tensor network analysis of critical coupling in two dimensional 4 theory, J. High Energy Phys. 05 (2019) 184.

[37] S.-J. Chang, Existence of a second-order phase transition in a two-dimensional $\varphi^{4}$ field theory, Phys. Rev. D 13, 2778 (1976).

[38] M. C. Bañuls, K. Cichy, J. I. Cirac, and K. Jansen, The mass spectrum of the Schwinger model with matrix product states, J. High Energy Phys. 11 (2013) 158.

[39] A. Milsted, J. Haegeman, and T. J. Osborne, Matrix product states and variational methods applied to critical quantum field theory, Phys. Rev. D 88, 085030 (2013).

[40] B. Buyens, J. Haegeman, K. Van Acoleyen, H. Verschelde, and F. Verstraete, Matrix Product States for Gauge Field Theories, Phys. Rev. Lett. 113, 091601 (2014).

[41] D. Schaich and W. Loinaz, Improved lattice measurement of the critical coupling in $\phi_{2}^{4}$ theory, Phys. Rev. D 79, 056008 (2009).

[42] P. Bosetti, B. De Palma, and M. Guagnelli, Monte carlo determination of the critical coupling in $\phi_{2}^{4}$ theory, Phys. Rev. D 92, 034509 (2015).

[43] S. Bronzin, B. De Palma, and M. Guagnelli, New monte carlo determination of the critical coupling in $\phi_{2}^{4}$ theory, Phys. Rev. D 99, 034508 (2019).

[44] S. Rychkov and L. G. Vitale, Hamiltonian truncation study of the $\varphi^{4}$ theory in two dimensions, Phys. Rev. D 91, 085011 (2015).

[45] T. Sugihara, Density matrix renormalization group in a twodimensional $\lambda \phi 4$ Hamiltonian lattice model, J. High Energy Phys. 05 (2004) 007. 